\title{
Anomalous Seismicity Patterns Study of Three Mainshocks Occurred in Arakan-yoma Region
}

\author{
Ananta Panthi \\ Department of Physics, Butwal Multiple Campus, Butwal, Nepal \\ apanthi@hotmail.com
}

\begin{abstract}
The anomalous seismic activity prior to three mainshocks occurred in Arakan-yoma region, within the area bounded by $20^{\circ}-28^{\circ} \mathrm{N}$ and $92^{\circ}-98^{\circ} \mathrm{E}$, has been studied considering spatial distribution and temporal patterns of earthquakes. The anomalous seismic activity has been investigated in this region using the seismicity data for the period 1963 to 2008 with events having magnitude $m_{b} \geq 4$.3. From the study, it has been inferred that all three mainshocks occurred in Arakan-yoma region were well preceded by anomalous seismicity pattern.
\end{abstract}

Keywords: anomalous seismicity, swarm, preparatory area, mainshock and precursory gap.

\section{INTRODUCTION}

The Indo-Burma region forms complex geology and tectonics. On the basis of physiographic structure, Burma territory has been divided as Eastern Highlands, Central lowlands and IndoBurma mountain belt (called Indo-Burma Range). Bengal basin lies to the west of Indo-Burma range. The Arakan-yoma, Manipur, Naga Hills, Lushai and Patkai are the most prominent structure in the Indo-Burma range. Burmese volcanic arc divides the Burmese low lands into eastern and western channels. The northernmost part of Indo-Burma arc joins with Himalayan belt and the joint region is outlined a complex structure that forms Syntaxis called Eastern Syntaxis. The Kabaw and Sagaing faults are the major faults in IBR.

The Northeast India and the Eastern segment of the Himalaya is seismically one of the most active regions in the world where several large and two great earthquakes have occurred during the past hundred years. Several thrusts, lineaments, folded belts are found to be responsible for the earthquake generation in this region. The high seismic activity in the region is due to northward as well as eastward movement of the Indian plate toward the Eurasia and Burma plates respectively [1, 2, 3, 4].

The seismicity fluctuation in space, time and depth are the common seismic precursor which plays an important role in identifying the anomalous seismic activity preceding moderate to large size earthquakes.
The anomalous seismic activities are characterized by intense seismic activity in the preparatory area as compared to the activities in the phases preceding and following it. The seismic activity prior to mainshock in the preparatory area can be classified into three episodes: the background, anomalous, and gap/ quiescence which are associated with low, high and low pattern of seismic activity, respectively [5]. The time of occurrence and the magnitude of the mainshocks are related with the magnitude of the events in the anomalous sequence, and the mainshock occurs after breaking the quiescence phase.

Several researchers have worked for the identification of precursory swarm sequence that precedes the major earthquakes occurred in northeast India region: Gupta and Singh [6], Singh et al. [7] and Shanker et al. [8] for north-east India; Singh and Singh [9] for north-east India and Pamir; Singh et al., [10] for Burma-Szechwan region. In the present study such phenomenon has been investigated prior to all the moderate size earthquakes that occurred from 1963 to 2008 in Arakan-yoma region; and a total of three such mainshocks were found preceding with such anomalous seismicity pattern.

\section{METHODOLOGY AND ANALYSIS}

The study of regional seismicity rate is an important factor in identifying the seismicity pattern in a region. The magnitude, frequency and focal depth are the major parameter to assess the seismicity pattern 
in a region. Identification of earthquake swarm/ anomalous seismicity phases preceding major earthquakes, in the present study, is based on spatial, temporal, magnitude and focal depth distributions and their relationships with the mainshocks.

In the present study, the earthquake data compiled from different available earthquake catalogues and prepared in appropriate computer format removing repeated events for the period 1963-2008. Cutoff magnitude was determined. The anomalous seismic activity, in this region, was investigated by using the seismicity data for the period 1963-2008 with $\mathrm{m}_{\mathrm{b}} \geq 4.3$ (cutoff magnitude). The preliminary investigations indicate that, three mainshocks in Arakan-yoma region were preceded by anomalous seismicity patterns for the period 1900 to 2000 .

\section{MAINSHOCKS}

The main three anomalous seismicity patterns occured in arakan-yoma region are discussed below.

\subsection{Mainshock of 7 June $2000\left(m_{b} 6.2\right)$}

This earthquake is a moderate shallow focus event and located at the extreme northeast part of the Arakan-yoma region. This earthquake has occurred in intense shallow focus active zone of Arakanyoma region. In order to investigate anomalous seismicity patterns associated with this earthquake, the seismicity data from 1990 to 2000 has been used within a grid $25.8^{\circ}-27.8^{\circ} \mathrm{N}$ and $96.4^{\circ}-97.6^{\circ} \mathrm{E}$.

Table 1: The characteristics of the identified seismic phases within their respective preparatory areas preceding three moderate size earthquakes (1) 7 June 2000 (6.2); (2) 6 May 1995 (6.3) and (3) 29 May 1994 (6.1) in Arakan-yoma region.

\begin{tabular}{|l|l|r|r|l|l|}
\hline \multirow{2}{*}{\multicolumn{1}{|c|}{ Seismic episodes }} & \multicolumn{2}{|c|}{ Period of Episodes } & \multirow{2}{*}{$\begin{array}{c}\text { No. of } \\
\text { events }\end{array}$} & $\begin{array}{c}\text { Annual } \\
\text { frequency }\end{array}$ & $\begin{array}{l}\text { Focal depth } \\
\text { range (km) }\end{array}$ \\
\cline { 2 - 6 } $\begin{array}{l}\text { (1) Mainshock of } \\
\text { 7 June 2000 (6.2) }\end{array}$ & & & & & \\
\hline Normal/ background (N) & 03Nov. 1990 -17 Mar. 1995 & 1596 & 2 & 0.5 & $41-59$ \\
\hline Anomalous/ swarm (A) & 18 Mar. 1995-09 Nov. 1995 & 236 & 3 & 4.6 & $33-65$ \\
\hline Precursory gap (G) & 10 Nov. 1995-06 June 2000 & 1672 & 0 & 0.0 & - \\
\hline $\begin{array}{l}\text { Mainshock/ aftershock } \\
\text { sequence (M) }\end{array}$ & 7 June 2000 & - & - & - & 33 \\
\hline $\begin{array}{l}\text { (2) Mainshock of } \\
\text { 6 May 1995 (6.3) }\end{array}$ & & & & & \\
\hline Normal/ background (N) & 25 Mar. 1992- 28 Jan. 1994 & 675 & 4 & 2.1 & $100-131$ \\
\hline Anomalous/ swarm (A) & 29 Jan.1994 - 02 Nov. 1994 & 277 & 8 & 10.5 & $93-193$ \\
\hline Precursory gap (G) & 03 Nov. 1994- 05May 1995 & 185 & 1 & 1.9 & 136 \\
\hline $\begin{array}{l}\text { Mainshock/ aftershock } \\
\text { sequence (M) }\end{array}$ & 06 May1995- 13 Aug. 1995 & 99 & 1 & & $118-119$ \\
\hline $\begin{array}{l}\text { (3) Mainshock of } \\
\text { 29 May 1994 (6.1) }\end{array}$ & & & & & \\
\hline Normal/ background (N) & 03 Sep. 1987-14 Feb. 1988 & 165 & 1 & 2.2 & 61 \\
\hline Anomalous/ swarm (A) & 15 Feb. 1988-08 Dec. 1989 & 662 & 7 & 3.8 & $33-60$ \\
\hline Precursory gap (G) & 09 Dec. 1989-28 May 1994 & 1633 & 2 & 0.4 & $33-37$ \\
\hline $\begin{array}{l}\text { Mainshock/ aftershock } \\
\text { sequence (M) }\end{array}$ & 29 May 1994-25 Nov. 1994 & 180 & 0 & - & 40 \\
\hline
\end{tabular}


After studying spatial and temporal distribution of events in the selected grid (Fig. $1 \mathrm{a}(1), \mathrm{b}$ ), the probable preparatory area was delineated for the mainshock and classified the events into four epochs as given in Table 1-1. The delineated preparatory area orients along northeast-southwest in the direction of major thrusts in which swarms events are located to the half northern portion and the mainshock occurred near the southern boundary (Fig. 1 a (1)). In normal background period, two events have occurred in four years and five months duration within the preparatory area that provides extremely low seismicity rate of the order of one event every two years. During eight months period of anomalous seismicity phase, four events have occurred in quick succession of which three are confined within the preparatory area (Fig. $1 \mathrm{a}(1)$ in its northern half portion and it is marked by 9 -fold increase in annual frequency of events as compared to the preceding normal seismicity phase (Fig. 1 b). The high frequency anomalous phase continued till 09 November 1995 following which mildly active precursory gap phase initiated which continued till the occurrence of mainshock on 7 June 2000. During this phase, only one event occurred on 28 June 1999 which is located outside the delineated preparatory area. The duration of precursory gap period is about 5.5 years (from the date of termination of anomalous sequence on 09 November 1995 till 06 June 2000) and it manifest an extremely low annual frequency (Table 1-1) which is at least 9-fold less active than the anomalous phase, similar to the normal seismicity phase, and hence it is the quiescence period that existed prior to the mainshock. The mainshock occurred on 7 June 2000 after five and half years of quiescence period and is located in the southwest part of the preparatory area. There was no aftershock activity observed in and around the epicenter tract of this shock. In the present case, the annual frequency estimated for all the phases behaves in the order of low-high-lowhigh seismicity rates and collectively constitute a well defined pattern of anomalous seismicity. Further, since tectonic activities in different delineated anomalous phases are confined in a short range of focal depths 33-65 km (Fig. 1c) also supports that these are inter-related. It is also evident that the existence of low-high-low pattern prior to the mainshock has some causative bearing on the occurrence of earthquakes and these may be useful, if similar observations are made, for understanding future seismic hazardous in northeast India region. Thus based on spatial, temporal and focal depth clustering of events in different anomalous phases, it can be concluded that the mainshock of 7 June 2000 in Arakan-yoma region was preceded by a well defined anomalous seismic activity.

\subsection{Mainshock of 6 May $1995\left(m_{b} 6.3\right)$}

This is an intermediate mainshock located within the main intermediate earthquake zone of Arakanyoma region and very close to the 6 August 1988 mainshock. The major tectonic features are oriented in north-south direction in this sector. The anomalous seismicity pattern for this mainshock has been investigated considering intermediate events only using seismicity data from 1992 to 1995 within a grid of $24.2^{\circ}-25.4^{\circ} \mathrm{N}-94.4^{\circ}-95.8^{\circ} \mathrm{E}$ (Fig. 1a(2)).

Using the spatial and temporal distribution of all the events falling in the selected grid for the period 25 March 1992 to 13 August 1995, a zone is delineated which is elongated approximately in east-west direction (Fig. 1a(2), d). Eleven shallow focus events, that are located within the selected grid, have been excluded in the analysis of seismicity pattern. While analyzing the data, the occurrence of nine events were noticed in nine months duration (29 January to 02 November 1994) in quick succession of which eight events fall well within the delineated preparatory zone (Fig. 1-d). These anomalous events are almost aligned in east-west direction within the zone and a mainshock occurred on 06 May 1995 between the alignment and the northern boundary of the delineated preparatory zone. In this anomalous episode, the annual frequency is estimated to be extremely high of the order of ten and half events and it is much higher than the preceding normal seismicity (25 Mar. 1992- 28 Jan. 1994) and the following precursory gap (03 Nov. 1994- 05 May 1995) episodes (Table 1-2). 


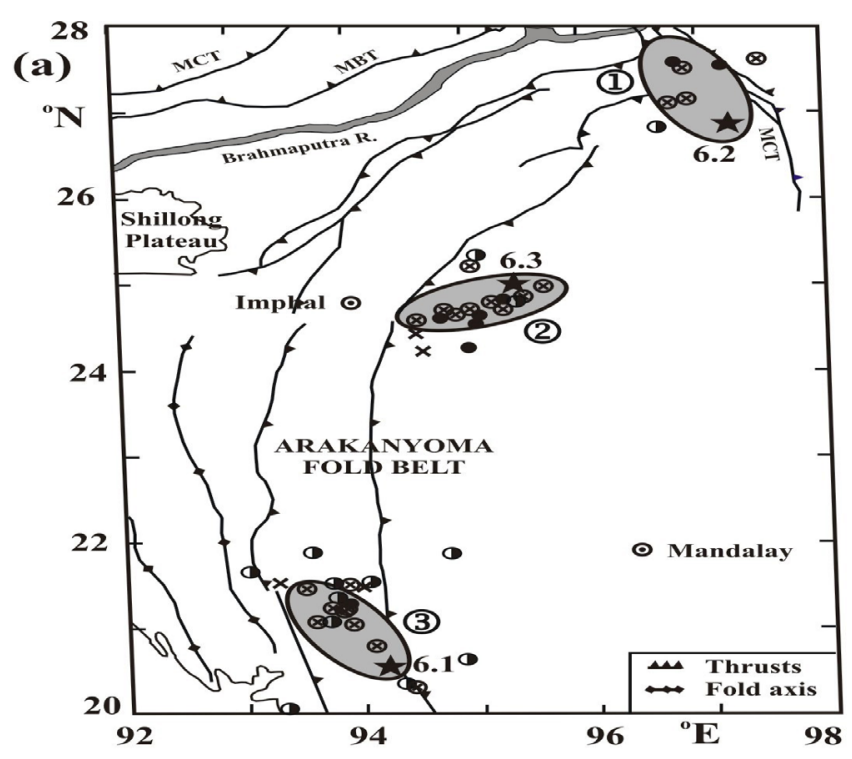

(b)
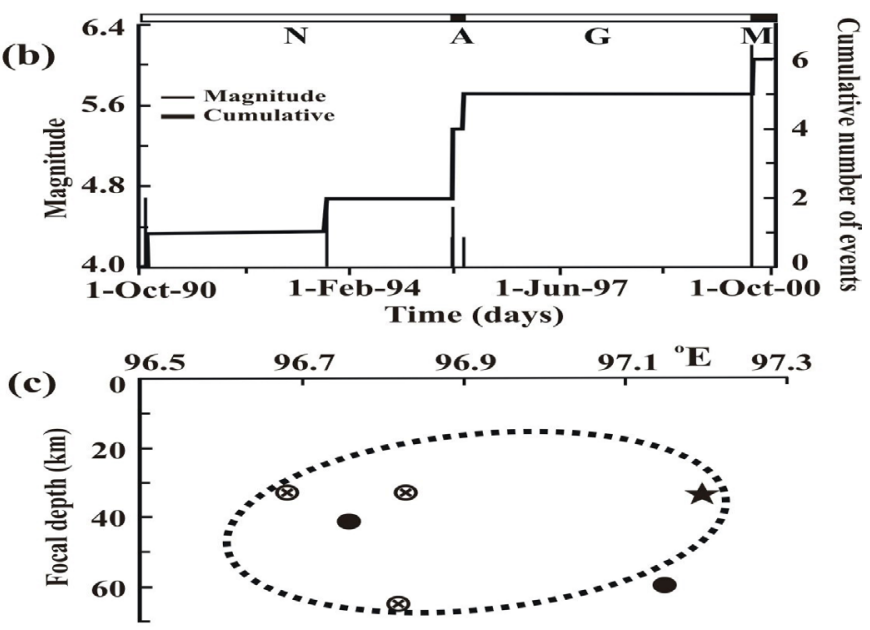

(d)
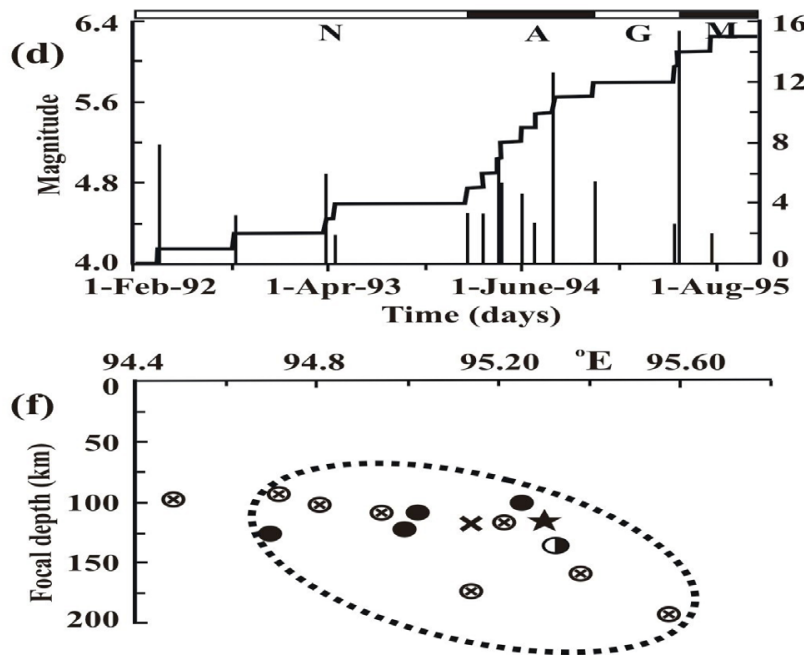

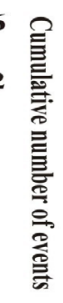

(e)
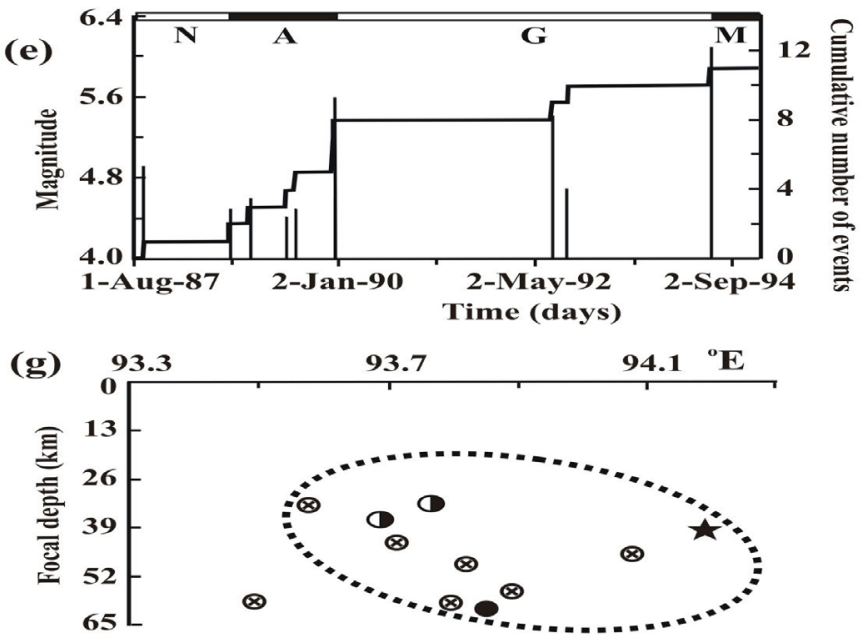

Fig. 1: Spatial $(a 1,2,3)$, temporal $(b, d, f)$ and focal depth $(c, e, g)$ distribution of earthquakes $\left(m_{b} \geq 4.3\right)$ prior to occurrence of three mainshocks of 7 June 2000 ( $m_{b}$ 6.2), 6 May $1995\left(m_{b}\right.$ 6.3) and 29 May 1994 ( $\left.m_{b} 6.1\right)$ in Arakan-yoma region. The solid and dotted elliptical areas are the preparatory zones delineated based on spatial and focal depth distribution of precursory events respectively.

It was estimated that 1.1:5.5:1 as the ratio of annual frequencies in normal, anomalous and precursory gap episodes respectively and these collectively constitute a well defined pattern of anomalous seismic activity for the mainshock of 06 May 1995. There was some short period aftershock activity in and around the preparatory zone. Moreover, the mainshock has occurred at a focal depth of 118 $\mathrm{km}$; whereas other events in normal, anomalous and gap periods in the focal depth range 93-193 km (Fig. 1-e). It is presumed that geological process that caused these events is also responsible for the mainshock occurrence especially in deep interiors keeping in mind the inherent errors in the focal depth estimation. The largest magnitudes of events in normal, anomalous and precursory gap episodes are observed to be 5.2, 5.9 and 4.4 respectively and the extent of the precursory gap period ( $\sim 6$ months only) is short for this magnitude mainshock. However, short precursory gap period may be attributed to the time variant internal geological processes as well as geologic setup of the region.

\subsection{Mainshock of 29 May $1994\left(m_{b} 6.1\right)$}

This is a shallow focus mainshock occurred at a depth of $40 \mathrm{~km}$ in the main intermediate zone of 
the Arakan-yoma tectonic belt and located in the southern portion of the study area (Fig. 1-a(3). To investigate the anomalous seismicity pattern associated with this mainshock, seismicity data from 1987 to 1994 has been considered in a grid bounded by $20.0^{\circ}-22.0^{\circ} \mathrm{N}$ and $93.0^{\circ}-95.0^{\circ} \mathrm{E}$ that encloses the epicenter of the mainshock.

During the specified period, nine events with intermediate depths have occurred which are excluded in the analysis. First of all, preparatory area for the mainshock was delineated based on spatial and temporal distribution of all the events occurred during 1987 to 1994 in the selected grid which is observed to elongate approximately in northwestsoutheast direction (Fig. 1-a (3), f). The general pattern of occurrence of events was observed and they were classified into four anomalous phases with their duration as: Normal/ background (03 Sep. 1987-14 Feb. 1988); Anomalous (15 Feb. 1988-08 Dec. 1989); Precursory gap (09 Dec. 1989-28 May 1994) and Mainshock and aftershocks (29 May 1994-25 Nov. 1994). The characteristics of events, annual frequencies and focal depth range in different identified episodes are given in Table 1-3. The time extent of normal, anomalous, precursory gap and mainshock-aftershock sequences are estimated as $0.5,1.8,4.5$ and 0.5 years respectively and the annual frequencies are 2.2, 3.8, 0.4 events in the first three episodes. During normal phase, only one event with magnitude $4.9(\mathrm{~h}=61 \mathrm{~km})$ occurred within the preparatory area. In the following anomalous phase, nine events occurred of which seven events fall within the preparatory area in the magnitude range 4.4-5.6 (focal depth range 33-60 km). This situation gives annual frequency of the order of 3.8 events which is higher by two-fold as compared to preceding normal episode. During the gap period, ten events occurred of which two events are located well within the preparatory area in the magnitude range 4.7-5.4 $(\mathrm{h}=33-37 \mathrm{~km})$ and the remaining are scattered outside around the periphery of the preparatory area. The annual frequency in the gap is estimated as one event every 2.5 years which is much lower (9-fold) than the preceding anomalous phase. The mainshock occurred on 29 May 1994 after 4.5 years of quiescence period with extremely low annual frequency. The temporal patterns and focal depth distribution of events in different episodes are depicted in Fig. 1-f, g. Similar to other two cases in Arakan-yoma region, low-highlow patterns of seismic activities are observed in different identified anomalous episodes preceding 29 May 1994 mainshock. These data suggest that a single tectonic process has been responsible for the generation of the events in the preceding episodes and also the mainshock of 29 May 1994.

\section{RESULTS AND DISCUSSION}

Various precursory phenomena are the indications of state of seismic energy build up in a weak zone along a fault which eventually leads to earthquakes. The simplest way to understand such indication is the seismicity patterns and it is possible to establish relation with forthcoming earthquake by using such observations. Medium to large earthquakes are known to precede epochs of abnormal seismicity patterns as background/normal seismicity, swarm/ anomalous seismicity, quiescence and foreshocks $[5,7,8,11,12]$.

In the present study, three mainshocks: 29 May 1994 (6.1), 6 May 1995 (6.3) and 7 June 2000 (6.2) were observed preceding well defined patterns of anomalous seismic activity in Arakan-yoma region. Moderate to extremely high annual frequencies (4 to 11 events per year) were observed in the identified anomalous seismic activity phases as compared to the preceding normal and the following gap periods in all investigated cases. Investigations carried out by several workers on anomalous seismic activity/ precursory swarms in different regions have reported that the mainshock magnitude is to be 1 to 2 units greater than the largest swarm event in the anomalous seismicity phases $[5,7,8,13]$. Here, this difference was observed as 0.4 to 1.2 in Arakan-yoma region. Also the focal depths of events in anomalous phase and the mainshocks were also observed within acceptable limit. These observations suggest that the events occurred at some time prior to the mainshocks showing clustering pattern in space and time domains constituted a well defined pattern of anomalous seismic activity.

The estimated precursory gap periods (measured from date of onset of anomalous phase to the time of occurrence of mainshocks) ranges from as low as 462 
days to 2295 days for all the magnitudes. It is evident from the data furnished in Table 1 that the logarithmic of precursory time period is directly proportional to the magnitude of the mainshocks. The precursory time period of 462 days is estimated for earthquake 6 May 1995 (6.3) and it is so low that it does not fit well with the behavior of long range earthquake precursory phenomena as pointed out by Rikitake [14]. However, spatial and temporal clustering, range of magnitudes and focal depths of the events forming the anomalous seismic activity prior to this mainshock have been established very well. It is visualized that some kind of high intensity geological phenomena coupled with the normal process might have resulted the mainshock at an early date.

\section{CONCLUSION}

From the study it is inferred that anomalous seismic activity exists prior to medium to large size earthquakes in Arakan-yoma region and there exist causal relationship between this pattern and the source parameters of the impending mainshocks. The information derived through such patterns can be employed for long-range earthquake prediction.

\section{ACKNOWLEDGEMENT}

The author is thankful to Professor Dr. H. N. Singh, BHU Varanasi, for his full supervision in this research work

\section{REFERENCES}

[1] Acharyya, S. K., Mitra, N. D., and Nandy, D. R., 1986. Regional geology and tectonic setting of North-East India and adjoining region, Geol. Surv. India Memoirs, 119, 6-12.

[2] Curray, J. R., Emmel, F. J., Moore, D. G., and Raitt, R. W., Structure, 1982. tectonics and geological history of the northeastern Indian Ocean. In: The Ocean Basins and Margins, 6, (eds.), Nairn, A. E. and Stehli F. G.,. Pub. Plenum Publishing Corporation, 399-450.

[3] Evison, F. F., 1977. Fluctuation of seismicity beforemajorearthquakes, Nature, 266,710-712.

[4] Gupta, H. K., and Singh, H. N., 1989. Earthquake swarms precursory to moderate to great earthquakes in the northeast India region, Tectonophysics, 167, 285-298.
[5] Gupta, H. K., and Singh, H. N., 1986. Seismicity of northeast India region: Part II: earthquake swarm precursory to moderate magnitude to great earthquakes, J. Geol. Soc. India 28, 367-406.

[6] Ni, James and Barazangi, M., 1984. Seismotectonics of the Himalayan collision zone: geometry of the underthrusting Indian plate beneath the Himalaya, Jour. Geophys. Res., 89, 1147-1163.

[7] Mitchel, A. H. G., 1981.Phanerozoic plate boundaries in mainland SE Asia, the Himalayas and Tibet, Jour. Geol. Soc. Lond., 138, 109-122.

[8] Paudyal, H., and Singh, H. N., 2007. Anomalous seismic activity in far western Nepal and its adjoining regions, Proc. National seminar on Modern Trend in Geophysical Sciences and Techniques, Indian School of Mines University, Dhanbad, 40-43.

[9] Rikitake,T., 1979.Classificationofearthquake precursors, Tectonics, 54, 293-309.

[10] Singh, H. N., Shanker D., and Singh, V. P., 2005. Occurrence of anomalous seismic activity preceding large to great earthquakes in northeast India region with special reference to 06 August 1988, Phys. Earth and Planet. Inter., 148, 561-284.

[11] Shanker, D., Singh, H. N., Paudyal, H., Kumar, A., Panthi, A., and Singh, V. P., 2010. Searching for an Earthquake Precursor- A case study of precursory swarm as a real seismic pattern before Major Shocks, Pure and Appl. Geophys., 167, 655-666.

[12] Singh, V. P., and Singh, H. N., 1986. Swarm hypothesis for occurrence of medium size earthquakes, Earthquake Prediction Res., 4, 83-94.

[13] Singh, V. P., Singh, H. N., and Singh. J., 1982. On the possibilities of premonitory swarms for three sequences of Earthquakes of BurmaSzechwan region, Tectonophysics, 85, T21-T29. [14] Shankar, D., Singh, H. N., and Singh, V. P., 1995. Anomalous seismic activity and longrange earthquake prediction in Himachal Pradesh, India, Acta Geod. Geoph. Hung., 30 (2-4), 379-395. 